\title{
Editorial
}

\section{Requiem for Reuse of Single-Use Devices in US Hospitals}

\author{
Martin S. Favero, $\mathrm{PhD}$
}

The subject of reusing single-use devices (SUDs) is one that has been discussed, debated, dissected, promoted, and damned seemingly forever. I have been attending conferences and professional and scientific meetings that discussed the pros and cons of reusing SUDs for more than 25 years and, until recently, it was the same story.

Those who favored, and indeed practiced, reusing SUDs pointed out the substantial cost savings and the absence of records of adverse patient reactions. Those who opposed the practice listed many worrisome issues, especially for those devices that were complicated in design and function: cleaning and sterilization, infections and pyrogenic reactions, functionality and its validation, materials compatibility, germicide residue, patient consent, liability, ethics, and so on. The United States Food and Drug Administration (FDA) during those years stated that the Medical Device Amendments of 1976 of the Food, Drug, and Cosmetic Act gave the agency regulatory authority over the manufacture of medical devices but not their use. The FDA did not regulate medical practice, and those hospitals that chose to reuse SUDs were responsible for the device and accepted the liability for the device. The FDA expressed concern but did not recommend against the practice of reuse. ${ }^{1}$

In the year 2000 all of that changed. Within months, the FDA issued policy statements that were unprecedented and that, for all practical purposes, abolished the practice of reusing SUDs in US hospitals. Subsequent to a number of meetings sponsored by the FDA and the Association for the Advancement of Medical Instrumentation (AAMI), the FDA in February 2000, issued for public comment two draft guidelines for reprocessing SUDs and on August 2, 2000 , released a guidance document on enforcement for reprocessing of SUDs by hospitals or third parties. That document presented the FDA's intent to enforce premarket submission requirements within 6 months (February
2001) for class III devices (eg, cardiovascular intra-aortic balloon pump, transluminal coronary angioplasty catheter); 12 months (August 2001) for class II devices (eg, blood pressure cuff, bronchoscope biopsy forceps), and 18 months (February 2001) for class I devices (eg, disposable medical scissors, ophthalmic knife).

The FDA uses two types of premarket requirements for non-exempt class I and II devices: a $510(\mathrm{k})$ submission that must show that the reprocessed device is as safe and effective as the same device when new, and a premarket approval application (PMA). The $510(\mathrm{k})$ submission must provide scientific evidence that the device is safe and effective for its intended use. The FDA is giving hospitals a year to comply with the non-premarket requirements (registration and listing, reporting adverse events associated with medical devices, quality system regulations, and proper labeling). Thus, if a hospital (or third-party reprocessor) is reprocessing devices intended for single use, then it must comply with the federal controls that apply to a new manufacturer of the medical device. For hospitals, the options are to stop reprocessing SUDs, comply with the rule, or outsource to a third-party reprocessor. The FDA guidance document does not apply to permanently implantable pacemakers, hemodialyzers, opened but unused SUDs, or healthcare facilities other than hospitals.

The FDA states that SUDs are medical devices, and hospitals or third parties that reprocess SUDs will be considered "manufacturers" and will be regulated in the same manner as the original equipment manufacturer. ${ }^{2}$ On April 23,2001 , the FDA sent a letter to hospital administrators and risk managers indicating that enforcement would begin by August 14, 2001. The FDA does not recommend against reusing SUDs, but because SUDs now must be reprocessed with the same quality assurance and safety criteria used by the original manufacture, and premarket clearance is needed for each device, the FDA's new policy

From Advanced Sterilization Products, Johnson \& Johnson, Irvine, California.

Address reprint requests to Martin S. Favero, PhD, Director, Scientific and Clinical Affairs, Advanced Sterilization Products, Johnson \& Johnson, 33 Technology Dr, Irvine, CA 92618.

O1-ED-11O. Favero MS. Requiem for reuse of single-use devices in US hospitals. Infect Control Hosp Epidemiol 2001;22:539-541. 
has virtually stopped the practice of reuse in US hospitals. Hospitals simply do not have the expertise to comply with these new regulations and, if they wish to have SUDs reprocessed, will have to rely on the dozen or so third-party reprocessors for that service. The FDA intends to inspect third parties directly. The agency will collaborate with the Joint Commission for the Accreditation of Healthcare Organizations (JCAHO) to help identify hospitals that have reuse programs. ${ }^{2}$ Hospitals that reprocess SUDs but do not comply with FDA regulations will be subject to fines of up to $\$ 1$ million per violation, and hospital staff involved with the reuse program may be prosecuted.

Time will tell if the third-party reprocessors can comply with the new regulations. In my opinion, they will not be able to comply for SUDs that are complex critical devices, such as cardiac catheters and ballooned instruments. This is not because the FDA standards are too restrictive, but because many reprocessed devices cannot and will not meet the standards that new devices are held to by the FDA. For pertinent FDA documents, see www.fda.gov/ cdrh/reuse/index.shtml.

How did we get from an era when any hospital could reuse SUDs to the present, where no hospital will dare do so? Is it just the recent FDA policy statements? I believe it is due to a number of developments that culminated in the FDA's involvement and subsequent actions.

Concern about the safety of reusing SUDs, especially complicated ones that penetrate mucous membranes or enter sterile areas of the body, has been increasing for years. More sophisticated studies are being done to examine the efficacy of the reprocessing techniques used. There has been a virtual renaissance in the science of medical device cleaning and the validation of such cleaning. The article published by Heeg and colleagues in this issue of the Journal is a good example. ${ }^{3}$ The objective of their study was to determine whether reprocessed SUDs would meet the international regulatory standards for sterility and meet the same materials standards as new devices. They tested reusable devices and SUDs. They found that all the devices could not be completely cleaned, but that SUDs tended to be more difficult to clean than reusable devices; that reusable devices could be disinfected adequately and SUDs could not; and that SUDs suffered materials destruction during the reprocessing.

From a historical point, the first disposables on the hospital market were constructed from inexpensive, heatsensitive materials such as plastics. They were usually manufactured outside the hospital and were purchased by the hospital from the supplier, presterilized and prepackaged. The initial unit cost was very low, and it usually did not make economic sense to collect these devices after use and reprocess them. Rather, they were purchased with the intent to be used once and then discarded; thus, they were labeled as disposable. ${ }^{4}$ In the 1970 s there was a revolution in medical biotechnology that permanently altered the nature of the medical-surgical device industry. New instruments and devices became available rapidly. They were sophisticated and complex and had electronic circuitry, membrane technology, expensive optics, and miniaturized components. It has been estimated that more than two thirds of the thousands of sterile devices used daily in American hospitals are purchased from industry, prepackaged, presterilized, and intended for one use only. ${ }^{5}$ They are called disposables, but they are certainly a far cry from the traditional cotton swabs, surgical sponges, and plastic bedpans that share that appellation. ${ }^{4}$

These devices are made of materials that usually require low-temperature sterilization by radiation, ethylene oxide gas, or hydrogen peroxide gas plasma. The vast majority of single-use items are not designed for reuse. The original raw materials used in the manufacture of SUDs may not be strong enough to provide reliable service more than once. Also, SUDs have not been designed for the cleaning and sterilizing technology employed by the hospital's central sterilization department. This technology might be ideal for reusable devices but quite incompatible with more complex and sophisticated SUDs.

The Centers for Disease Control and Prevention (CDC), in its 1981 "Guideline for the Prevention and Control of Nosocomial Infections (Guidelines for Environmental Control)," provided a clear and categorical recommendation against reprocessing of devices that are meant for one-time use. ${ }^{6} \mathrm{~A}$ few years later, the CDC removed the recommendation against reusing SUDs. It was stated that, because there was a lack of evidence indicating increased risk of nosocomial infection associated with the use of all SUDs, a categorical recommendation against reusing all types of SUDs was not justifiable. ${ }^{7}$

Another reason a categorical recommendation could not be made by the CDC was that the reuse of hemodialyzers (artificial kidneys) was becoming a standard of practice. The hemodialyzer was an SUD that was reprocessed and reused on the same patient. In the 1970 s, approximately $18 \%$ of hemodialysis centers reported reusing dialyzers. However, in 1982 the Health Care Financing Administration (HCFA), which fully pays for end-stage renal disease, including hemodialysis treatments, substantially reduced the amount of money paid to centers for treating patients. Up until that time, the reuse of hemodialyzers was done because patients seemed to feel better when they were dialyzed with reused dialyzers compared to new ones. ${ }^{8}$ During 1976 to 1996 , the percentage of dialysis centers in the United States that reported reuse of disposable hollow-fiber dialyzers increased from $18 \%$ to $84 \%$. 910

In the mid-1980s there was public and patient concern expressed about the safety of reusing dialyzers, and the US Public Health Service (PHS) undertook an investigation of the practice of dialyzer reuse in US hemodialysis centers. The PHS concluded that the practice of reusing dialyzers with the same patient appeared to be safe if performed according to strict and established protocols. In 1986, the PHS (the major agencies were the FDA, the CDC, and the National Institutes of Health) subsumed the AAMI's guidelines for reusing hemodialyzers ${ }^{11}$ and recommended these as PHS guidance to HCFA, which, in turn, made them conditions for participation in Medicare and Medicaid. In short, 
the AAMI guidelines were adopted as PHS guidance and later became HCFA regulations. Some years later, the FDA directed manufacturers of dialyzers to insert on the labels of these devices instructions for reusing them-in spite of the fact that all dialyzers at that time were labeled for one-time use. The FDA's rationale was that the manufacturers were aware the devices were being reused and therefore had the responsibility for providing instructions for reuse. The manufacturers ultimately cited the AAMI standards on the dialyzer labels to be used as methods of reuse.

In general, dialyzer reuse does appear to be safe if performed according to strict and established protocols. In CDC surveys, dialyzer reuse has not been associated with infection with hepatitis $B$ virus or hepatitis $C$ virus, but it has been associated with pyrogenic reactions in most years since $1989^{9,12}$ and has been linked to a number of outbreaks of pyrogenic reactions, bloodstream infections, and nontuberculous mycobacterial infections. The outbreaks associated with dialyzer reuse have been due to user errors involving breaches in the reprocessing protocol, such as use of incorrect concentrations or application of chemical germicides or the failure to maintain standards for water quality. ${ }^{8}$ Manual reprocessing of dialyzers that does not include a test for membrane integrity, such as the air-pressure leak test, may fail to detect membrane defects and be a cause of pyrogenic reactions. ${ }^{13}$ When Julie Garner and I wrote the section on reuse in the 1985 CDC "Guidelines for Handwashing and Hospital Environmental Control," we worried that hospitals might interpret the removing of the recommendations against reuse of SUDs as a signal that reuse had little or no risk. Consequently, we inserted into the guideline relatively conservative recommendations that we thought would discourage the reuse of critical and complicated SUDs?:

- Items or devices that cannot be cleaned and sterilized or disinfected without altering their physical integrity of function should not be reprocessed.

- Reprocessing procedures that result in residual toxicity or compromise the overall safety or effectiveness of the items or devices should be avoided.

In addition, we referenced the report from an international conference on reuse of SUDs, where arguments for and against reuse were discussed. ${ }^{14}$ We believed at the time that these constraints were such that very few, if any, hospitals would reprocess complicated high-risk SUDs. We did not anticipate that years later hospitals not only would be reprocessing complicated high-risk SUDs of all types but also that there were no standardized protocols for this practice and that patients, as well as physicians, were not being informed of this practice-not to mention all of the controversy over ethical and legal issues.

The debate on the subject that occurred among healthcare professionals and the infection control community eventually spilled over into the public arena. After much public pressure and the interest of Congress, the FDA took action in an uncharacteristically swift manner.

A number of European countries have already reached the point of banning reuse of SUDs, and I would anticipate that a number of other countries will follow their and the US's path.

Is reuse really gone? I believe it is gone from hospitals. As mentioned above, hospitals cannot comply with the FDA regulations on reuse. Hospital administrators, risk managers, and legal advisors will not allow this practice to occur. Whether they will allow in their hospitals the reuse of SUDs reprocessed by third parties is unknown. The ethical and legal issues remain the same, and the JCAHO will be identifying hospitals with reuse programs involving third parties.

How and if the third-party reprocessors can comply with the FDA regulations is unclear. The submission of materials for premarket clearance is no easy task. The more complex the device, the more likely it is that the amount of effort involved for a successful submission will be so Herculean that it may be impossible to actually receive FDA clearance. This is not because the FDA standards are too restrictive, but because many reprocessed devices cannot and will not meet the standards that new devices are held to by the FDA. Some SUDs that are class I devices may stand a chance of receiving clearance; class II devices are anybody's guess.

The requiem and the wake for reuse of SUDs in hospitals have passed. There will not be a resurrection.

\section{REFERENCES}

1. Villforth JC. Position of the US Food and Drug Administration. In: Proceedings of an International Conference: Reuse of Disposable Medical Devices in the 1980s. March 29-30, 1984; Washington, DC. Washington, DC: Georgetown University Medical Center; 1984:91-94.

2. Ulatowski TA Reuse of single-use devices In: Rutala W, ed. Disinfection, Sterilization and Antisepsis. Washington, DC: Association for Professionals in Infection Control and Epidemiology; 2001:16-27, 93.

3. Heeg P, Roth K, Reichl R, Cogdill CP, Bond WW. Decontaminated single-use devices: an oxymoron that may be placing your patients at risk for crosscontamination. Infect Control Hosp Epidemiol 2001;22:542-549.

4. Greene WV. Reuse of disposable devices. In: Mayhall CG, ed. Hospital Epidemiology and Infection Control. Philadelphia, PA: Lippincott Williams \& Wilkins; 1999:1201-1208.

5. Bruch CW. Inhospital Versus Industrial Sterility Assurance: Is There a Double Standard? Inhospital Sterility Assurance: Current Perspectives. Arlington, VA: Association for the Advancement of Medical Instrumentation; 1982:19-22. Technology Assessment Report 4-82.

6. Favero M. Position of the Centers for Disease Control. In: Proceedings of an International Conference: Reuse of Disposable Medical Devices in the 1980s. March 29-30, 1984; Washington, DC: Georgetown University Medical Center, 1984:98-101.

7. Garner JS, Favero MS. Guidelines for handwashing and hospital environmental control, 1985. Am J Infect Control 1986;14:110-126.

8. Favero, MS, 'Tokars JI, Arduino MJ, Alter MJ Nosocomial infections associated with hemodialysis. In: Mayhall CG, ed. Hospital Epidemiology and Infection Control. 2nd ed. Philadelphia, PA: Lippincott Williams \& Wilkins; 1999:897-917.

9. Tokars JI, Miller ER, Alter MJ, Arduino MJ. National surveillance of dialysis associated diseases in the United States, 1995. ASAIO J 1998;44:98-107.

10. Tokars JI, Miller ER, Alter MJ, Arduino MJ. National surveillance of dialysis associated diseases in the United States, 1997. Semin Dial 2000;13:75-85.

11. Association for the Advancement of Medical Instrumentation (AAMI) and American National Standards Institute (ANSI). American National Standard: Reuse of Hemodialyzers. Arlington, VA: Association for the Advancement of Medical Instrumentation; 1993. ANSI/AAMI RD47-1993.

12. Favero MS, Deane N, Leger RT, Sosin AE. Effect of multiple use of dialyzers on the hepatitis B incidence in patients and staff. JAMA 1981;245:166-167.

13. Bland L, Alter M, Favero M, Carson L, Cusick I. Hemodialyzer reuse: practices in the United States and implication for infection control. Trans Am Soc Artif Intern Organs 1985;31:556-559.

14. Institute of Health Policy Analysis. Proceedings of an International Conference: Reuse of Disposable Medical Devices in the 1980s. March 2930, 1984; Washington, DC. Washington, DC: Georgetown University Medical Center, 1984:91-94. 